\title{
LONG-TERM FACIAL NERVE CLINICAL EVALUATION FOLLOWING VESTIBULAR SCHWANNOMA SURGERY
}

\author{
Rafaela Julia Batista Veronezi', Yvens Barbosa Fernandes ${ }^{2}$, Guilherme Borges ${ }^{3}$, Ricardo Ramina ${ }^{2}$
}

\begin{abstract}
Background: Facial function is important in accompaniment of patients operated on vestibular schwannoma (VS). Objetive: To evaluate long term facial nerve function in patients undergoing VS resection and to correlate tumor size and facial function in a long-term follow-up. Method: Transversal study of 20 patients with VS operated by the retrosigmoid approach. House-Brackmann Scale was used preoperatively, immediately after surgery and in a long-term follow-up. Student $t$ test was applied for statistic analysis. Results: In the immediate postoperative evaluation, $65 \%$ of patients presented FP of different grades. Improvement of facial nerve function (at least of one grade) occurred in $53 \%$ in the long-term follow-up. There was statistically significant difference in facial nerve outcome in long-term follow-up when tumor size was considered $(p<0.05)$. Conclusion: The majority of patients had improvement of FP in a long-term follow-up and tumor size was detected to be a factor associated with the postoperative prognostic.
\end{abstract}

KEY WORDS: vestibular schwannoma, facial nerve function, retrosigmoid approach.

\section{Avaliação clínica tardia do nervo facial no pós-operatório de schwannoma vestibular}

Resumo - Contexto: A função facial é importante para acompanhamento dos pacientes operados de schwannoma vestibular (SV). Objetivo: Avaliar o grau de paralisia facial (PF) em pacientes operados de SV, correlacionando tamanho do tumor com função facial na avaliação tardia. Método: Estudo transversal com análise seriada de 20 pacientes com SV operados pela via retrosigmóide-transmeatal. A Escala de House-Brackmann foi utilizada no pré-operatório, pósoperatório imediato e pós-operatório tardio. $O$ teste $t$ de Student foi aplicado para análise estatística. Resultados: No pós-operatório imediato, $65 \%$ dos pacientes apresentaram graus variados de $\mathrm{PF}$, sendo que $53 \%$ destes obtiveram melhora de pelo menos um grau de House-Brackmann na avaliação tardia. Houve diferença significativa no resultado da função facial no pós-operatório tardio quando o tamanho do tumor foi considerado $(p<0.05)$. Conclusão: A maioria dos pacientes da amostra apresentou melhora da PF no pós-operatório tardio, sendo o tamanho do tumor um fator associado ao prognóstico.

PALAVRAS-CHAVE: schwannoma vestibular, função facial, via retrosigmóide.

Vestibular schwannoma (VS) is a benign tumor that arises from the eighth cranial nerve. It represents $8 \%$ to $10 \%$ of all brain tumors and $80 \%$ of all cerebellopontine angle tumors'. In 1894, Charles Balance performed the first successful VS excision, but the mortality rate at that time was devastatingly high ${ }^{2}$. Harvey Cushing improved surgical techniques and brought the mortality rate down to approximately $20 \%{ }^{3}$ performing sub-total removal of the tumors. Improvement in surgical techniques and development of new technological devices had brought the mortality rate to under $2 \%$. The advances and improvements in microsurgical techniques have changed the actual goals of surgery, being facial nerve preservation an utmost con- cern ${ }^{3}$. Paralysis of the facial expression muscles is a debilitating and psychologically devastating condition for the patient. Preservation of facial nerve function after VS surgery is one of the most important goals to be achieved.

There is no established method that allows precise prediction of the long-term prognosis of facial nerve palsy after VS surgery ${ }^{6-8}$. It has been suggested that tumor size is an approximate guide to predicting ultimate functional outcome $e^{9,10}$.

The objective of this study is to analyze the facial nerve function in patients submitted to VS surgery by the retrosigmoid transmeatal approach and correlate tumor size with the grade of postoperative facial palsy (FP).

\footnotetext{
'Physiotherapist, Master Degree in Medical Sciences for the Department of Neurology, State University of Campinas (UNICAMP), Campinas SP, Brazil; ${ }^{2}$ Assistant Professor, Department of Neurology, UNICAMP; ${ }^{3}$ Associate Professor, Department of Neurology, UNICAMP. This study was partially sponsored by CAPES (Coordenação de Aperfeiçoamento de Pessoal de Nivel Superior), Brazil.
}

Received 8 October 2007. Accepted 21 February 2008.

Dra. Rafaela Júlia Batista Veronezi - Rua Santarém Qd 235 Lt 08 / 802 Pq Amazônia - 74835-170 Goiânia GO - Brasil. E-mail: veronezi@fcm.unicamp.br 


\section{METHOD}

Subjects

Twenty patients were enclosed in this study. They underwent surgical resection of VS from January 1999 to October 2002 at the Hospital das Clínicas of the State University of Campinas (HC/UNICAMP), in Campinas, Brazil.

The Ethics Committee on Research, State University of Campinas (UNICAMP) - Brazil, approved the study and a free and informed consent was obtained from each participant.

\section{Procedures}

All patients were evaluated before surgery by computerized tomography (CT) and/or magnetic resonance (MR). Tumor size was considered as the largest extrameatal diameter. Tumors were categorized as small $(\leq 2.0 \mathrm{~cm})$, medium $(2.1-4.0 \mathrm{~cm})$ and large $(>4.0 \mathrm{~cm})$.

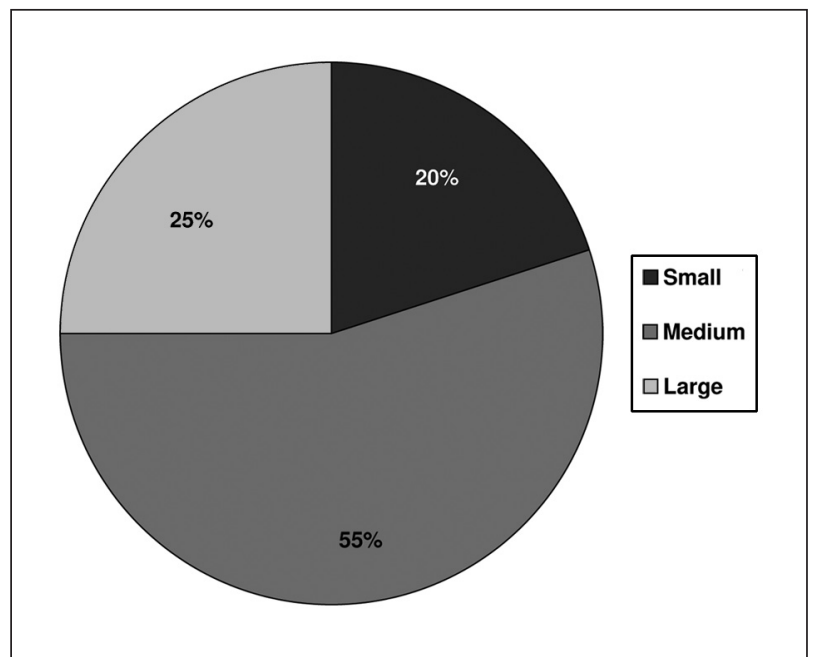

Fig 1. Vestibular schwannoma tumor size distribution (\%) in the 20 patients of the study, considering the largest extrameatal diameter. Tumors were categorized as small $(\leq 2.0 \mathrm{~cm})$, medium $(2.1-4.0 \mathrm{~cm})$ and large $(>4.0 \mathrm{~cm})$.
The surgical standard retrosigmoid transmeatal approach was used in all patients by the same surgical team. The main goals of treatment were total removal of the tumor without major morbidity and excellent preservation of facial nerve function. No patients were submitted to previous treatment of their tumors.

Facial function was assessed in three specified time intervals: preoperative, immediate postoperative ( 24 hours) and in a long-term follow-up (18 months or longer). It was reported using the House-Brackmann facial nerve function grading system ". Patients were divided into three subgroups: good facial function (Grades I-II), regular facial function (Grades III-IV), and poor facial function (Grades V-VI).

\section{Data analysis}

A descriptive analysis was performed, as well as statistical analysis using the Student $t$ test for independent data. Statistical significance was set at $p<0.05$ to determine the possible association of the tumor size on final facial function.

\section{RESULTS}

Fifteen patients were female $(75 \%)$ and five were male (25\%). Patients' ages ranged from 17 to 77 years old (mean 51 years old). Most patients ( $n=14)$ were older than 40 years old; $40 \%$ of patients $(n=8)$ were 40 to 60 years old and $30 \%(n=6)$ were older than 60 years old. There was no predominance regarding the side of the tumor. The mean tumor size was $3.38 \mathrm{~cm}$ with a range of $1.5 \mathrm{~cm}$ to $5.0 \mathrm{~cm}$. The distribution of the tumor size is represented in Fig 1. The minimum follow-up was 19 months and the longest one was 5 years and 10 months (mean time of 3 years and 10 months).

The evolution of facial nerve function in the three specified time span is illustrated in Fig 2. Three patients (15\%) had FP preoperatively. Two of them had HouseBrackmann grade $\mathrm{VI}$ in this period and the other one had

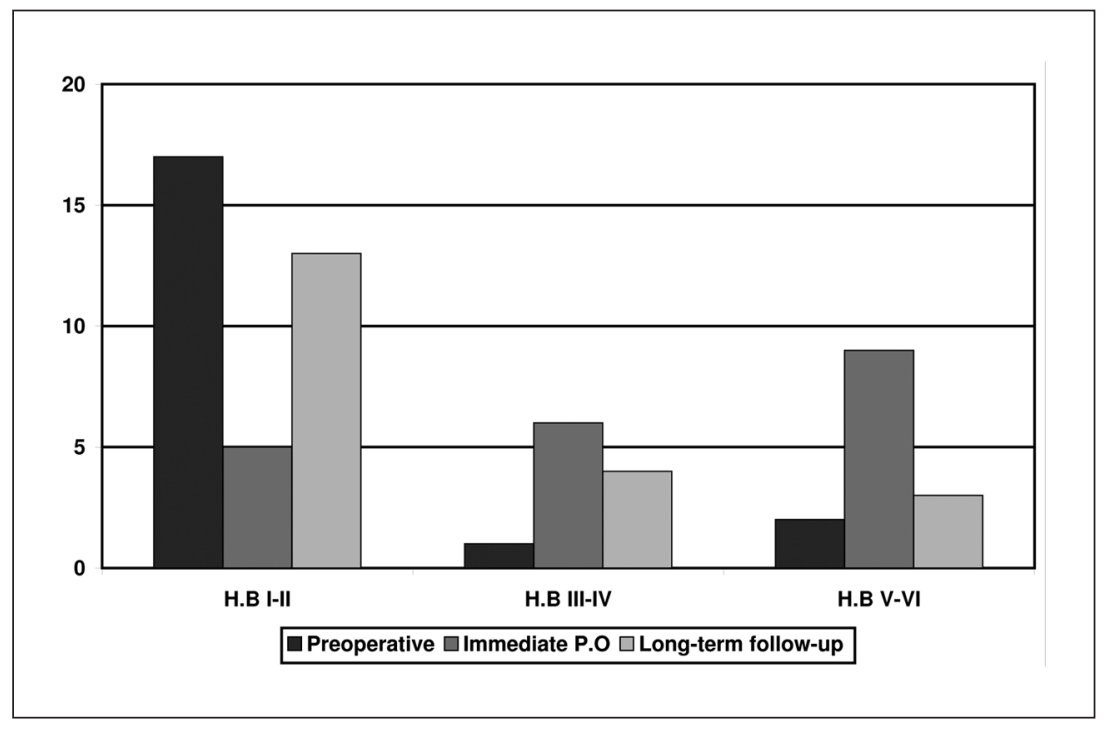

Fig 2. Evolution of facial nerve function according to House-Brackmann Scale in the three time span: preoperative, immediate postoperative and in a long-term follow-up (18 months or longer postoperatively). 


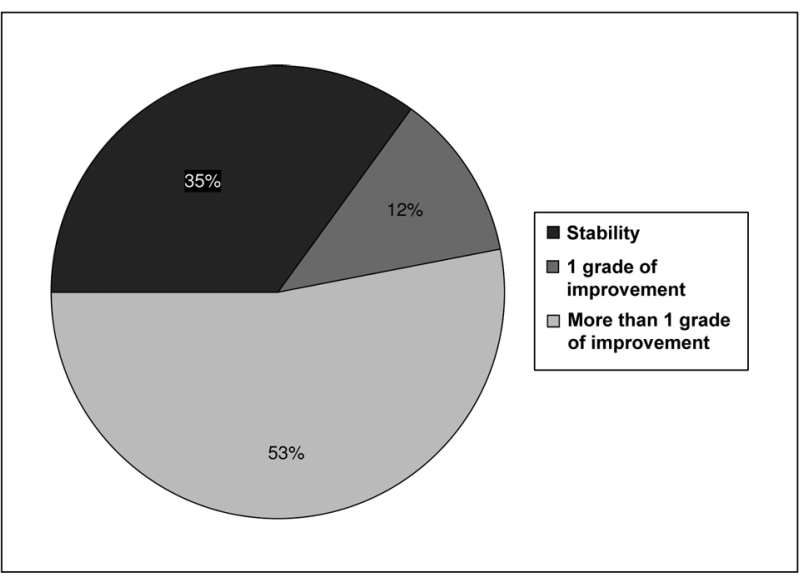

Fig 3. Evaluation of facial function in the long-term follow-up related to immediate postoperative time.

House-Brackmann grade III. These patients harbored medium or large size tumors.

The facial nerve could be anatomically preserved in 17 patients (85\%). A cable graft of sural nerve was performed to bridge the divided facial nerve in patients that the direct suture of the facial nerve was not possible.

A partial-to-total FP (Grades II-VI) was observed in the immediate postoperative course in 17 cases (85\%). Nine patients (45\%) presented poor facial function (Grades V$\mathrm{VI}$ ) postoperatively, including the 3 patients who already presented FP preoperatively.

In the majority of the cases FP improved in a minimum period of 18 months (Figs 2 and 3). In $30 \%$ of the cases ( $n=6)$, total restoration of facial nerve function (Grade I) was observed in the long-term follow-up. In $35 \%$ ( $n=7)$, a minimal FP (Grade II) remained. The facial nerve function was classified as good in 13 patients $(65 \%)$, regular in 4 patients $(20 \%)$ and poor in $3(15 \%)$. The patients with long-term poor facial function (Grade V-VI) presented FP preoperatively.
A hypoglossal-facial nerve anastomosis ${ }^{12}$ was carried out during the first year postoperative in 4 patients (20\%) that presented no recovery of the facial function. A House-Brackmann grade IV was obtained in 1 case, and grade III in 2 cases. One patient (tumor size $5.0 \mathrm{~cm}$ and previous FP) showed no improvement of FP. For this patient plastic surgery procedures are recommended for facial function rehabilitation.

No impairment of facial nerve deficit was observed in the interval between hospital discharge and follow-up examination.

Facial function in long-term follow-up was also analysed with respect to tumor size (Table 1). Patients with regular (Grades III-IV) or poor (Grades V-VI) facial function had medium and large tumors, respectively.

There was a statistically significant difference (Table 2) in immediate and long-term facial nerve outcome when the tumor size was considered. Patients who presented improvement of FP had smaller tumors.

\section{DISCUSSION}

VS is considered by many surgeons to be one of the most difficult brain tumors to be removed without additional neurologic deficits. Since the first successful resection of a VS occurred in $1894^{2}$, surgical techniques have been continuously refined to reduce patient morbidity and mortality rates ${ }^{3,4}$. Over the last century, treatment of VS has undergone immense changes and the focus of surgery has improved from prolongation of patient's life towards preservation of cranial nerve function ${ }^{5}$, specially the seventh nerve.

Loss of facial nerve function is a debilitating and psychologically devastating condition ${ }^{13}$. Patients with FP may experience several limitations as difficulty to speech and

Table 1. Final House-Brackmann Grades according to tumor size.

\begin{tabular}{ccccc}
\hline House- & \multicolumn{4}{c}{ Tumor size } \\
\cline { 2 - 5 } Brackmann & $\leq 2.0 \mathrm{~cm}$ & $2.1 \mathrm{~cm}-4.0 \mathrm{~cm}$ & $>4.0 \mathrm{~cm}$ & $\begin{array}{c}\text { Total } \\
\text { grade }\end{array}$ \\
$\mathrm{n}(\%)$ & $\mathrm{n}(\%)$ & $\mathrm{n}(\%)$ & $(\mathrm{n}=20)(\%)$ \\
\hline I-II & $4(20)$ & $7(35)$ & $2(10)$ & $13(65)$ \\
III-IV & 0 & $4(20)$ & 0 & $4(20)$ \\
V-VI & 0 & 0 & $3(15)$ & $3(15)$ \\
\hline
\end{tabular}

Table 2. Mean and standard deviation of the tumor size classified for improvement/no-improvement in long-term follow-up related to immediate postoperative time.

\begin{tabular}{lcccc}
\hline Improvement & Tumor size $($ mean, $\mathrm{cm})$ & Standard deviation & $t^{*}$ & $\mathrm{p}$ \\
\hline Yes $(\mathrm{n}=11)$ & 2.90 & 0.78 & 2.355 & 0.030 \\
No $(\mathrm{n}=9)$ & 3.97 & 1.23 & & \\
\hline
\end{tabular}

*Student $t$ test for independent data. Statistical significance was set at $\mathrm{p}<0.05$. 
eat and may experience drooling. Furthermore, these patients may have significant ophthalmic complications from loss of the blink reflex, upper and lower eyelid retraction, and lagophthalmos. The absence of orbicularis muscle tone also causes a loss of the corneal "squeegee" effect and predisposes the patient to dry eye symptoms and corneal exposure ${ }^{14}$. Socially, facial paralysis presents a distorted continence that others find disquieting. Affected individuals are avoided and often feel socially isolated. The combination of physical and psychological disabilities often results in a lasting postoperative depression.

The mean age of the patients of this series (51 years old) and the predominance of individuals of the feminine sex $(75 \%)$ were in agreement with studies that indicate greater frequency of the VS in females in the fifth and sixth decades of life $\mathrm{if}^{3,8,15-17}$.

Facial function results were reported by the HouseBrackmann facial nerve function grading system ${ }^{11}$. This allows for standardized comparisons of results, which is essential to valid medical decision making.

Translabyrinthine, retrosigmoid transmeatal, and middle fossa approaches are the three basic approaches for the removal of VS. However, recent papers have recommended the retrosigmoid transmeatal approach for removal of VS of all sizes with no mortality and minimal morbidity ${ }^{16-21}$. All VS surgeries in our unit were carried out by this approach that presents noteworthy advantages: short operating time, immediate and accurate identification of the tumor and facial nerve within the internal auditory canal (IAC), possibility of facial nerve reconstruction and control of the involved neurovascular structures. In addition, it allows a high rate of facial nerve function preservation because the facial nerve is identified within the IAC and at brain stem and is deeper than the tumor ${ }^{21}$.

According to current literature, $8 \%$ to $20 \%$ of patients undergoing VS surgery experience facial nerve injury, even in experienced hands, despite the use of intra-operative facial nerve monitoring ${ }^{22}$. In this series, the intra-operative facial nerve injury occurred in $15 \%$ of the cases. Moreover, the anatomical preservation of the facial nerve indicates no necessarily preservation of its function ${ }^{23}$.

The facial function immediately after VS surgery is extremely changeable, also being able to present itself without alteration. In our study, 3 patients (15\%) had no FP in this period, but the majority of the patients (85\%) presented some grade of facial nerve dysfunction (Grades II-VI). It can be caused by a number of possible mechanisms $^{24}$. The most common cause is direct trauma or nerve stretching during surgery. The facial nerve is often intimately involved with the tumor, resulting in its distortion through compression and stretching. The severity of ad- hesions between the tumor capsule and the facial nerve is highly variable ${ }^{15}$. Theoretically, both neuropraxia and axonotmesis are reversible phenomena and facial nerve function should fully return ${ }^{3}$. The involvement of the facial nerve with the tumor, either with the nerve passing through the tumor or with the tumor infiltrating the nerve sheath, was documented and graphically represented in the work by Sampath et al. ${ }^{25}$.

Another common mechanism of facial nerve injury is related to the vascular supply to the facial nerve. Maintaining the blood suplly to the facial nerve is critical. Thermal injury can also cause FP. It is usually transient, but occasionally it may lead to local vasoconstriction and cause secondary ischemic injury to the nerve ${ }^{3}$. These factors can modify its functional capacity and propitiate the collagen deposition ${ }^{26}$.

In the recent literature, the rate of good postoperative function of the facial nerve (Grades I-II) in 1 year or longer after surgery is reported to range from $70.7 \%$ to $96 \% \%^{10,16,21}$, if considering any approach whatever the tumor size. The result obtained in the present series was $65 \%$. However, the average dimension of the tumors of our series ( 3.38 $\mathrm{cm}$ ) was larger than the ones normally described in the literature ${ }^{6,8,10,15-17,20,23}$, and the percentage of medium and large tumors in our series was higher. This reflects a delayed diagnosis of the VS in our country. Axon et al..$^{15}$ reported a mean tumor size of $2.2 \mathrm{~cm}$, Fenton et al. ${ }^{6}$ of 2.0 $\mathrm{cm}$, and Arts et al. ${ }^{20}$ of $0.89 \mathrm{~cm}$. In the study of Zaouche et al. ${ }^{8}$ only $10.2 \%$ of patients had large tumors. The status of good facial nerve function when considering tumors extending more than $3.1 \mathrm{~cm}$ in the series of Wiet et al. ${ }^{10}$ was $61 \%$, a percentage very next to our index of $65 \%$.

The prognostic factors of postoperative FP in VS surgery have been variously reported ${ }^{6-8,27}$. Although tumor size alone should not be considered as a predictive factor, it has been thought to be the most important one ${ }^{9,10}$. Gormley ${ }^{28}$ showed that $96 \%$ of patients with small tumors had postoperatively normal facial function or slight dysfunction (Grade I-II), whereas only $38 \%$ of patients with tumors greater than $4.0 \mathrm{~cm}$ had no dysfunction. Mamikoglu et al. ${ }^{18}$ reported that $45 \%$ of patients with tumors greater than $3.0 \mathrm{~cm}$ had House-Brackmann grade I-II, 34\% had HouseBrackmann grade III-IV, and 20\% had House-Brackmann grade V-VI facial function at 1-year follow-up. Lanman et al. ${ }^{29}$ reported that $48 \%$ of patients with large tumors $(>3.0$ $\mathrm{cm}$ ) had poor facial nerve function at 1-year after surgery. Usually, favorable facial nerve function is achieved in an average of only $50 \%$ of patients with large VS operated on by the most experienced teams ${ }^{19}$. McElveen et al. ${ }^{23}$ showed that $56 \%$ of patients had House-Brackmann grade I-II at 1-year follow-up in tumors larger than $4.0 \mathrm{~cm}$. 
In our series tumor size proved to be an important variable on outcome in the long-term follow-up. Statistical significance $(\mathrm{p}<0.05)$ was demonstrated in comparison to immediate postoperative results, showing that a large tumor is associated with poorer postoperative facial nerve function (Table 2).

Facial nerve can tolerate a large degree of stretching, compression or distortion, which is caused by the tumor, without apparent FP. However, as the tumor grows, the individual fibers of the facial nerve may become splayed over the tumor capsule. Not surprisingly, very large tumors place the nerve under greater tension, which increases the likelihood of stretch injury and may explain the high rate of FP seen in patients with large tumors ${ }^{27}$. Alternatively, nerve dysfunction may result from poor vascularization of nerve segments that are effaced by large tumors.

It would appear that the bigger is the tumor the greater is the risk to the nerve. However, size cannot predict the relationship or invasiveness of the tumor with regard to the nerve, the degree of adhesiveness, or the difficulty of dissection ${ }^{6}$. The facial nerve can traverse any part of the tumor capsule and even pass through the tumor itself, and this occurs equally in small and large tumors ${ }^{25}$.

Patients with preoperative facial weakness had a poorer prognosis in terms of facial nerve function following VS surgery ${ }^{7}$. This also could be observed in our series. It may be due to infiltration of the facial nerve by the VS, especially if the tumor is large $\mathrm{e}^{30}$.

In conclusion, in spite of the large develpments in VS surgery in the last century, FP remains a frequent complication. The majority of the patients of this study presented improvement of the FP in an average time of 3 years and 10 months (65\%). It was clear that it is possible to obtain normal to near-normal facial function in patients operated on by retrosigmoid transmeatal approach. Tumor size was a significant factor for postoperative facial nerve function in a long-term follow-up. This is consistent with other published series.

\section{REFERENCES}

1. Kartush JM, Brackmann DE. Acoustic neuroma update. Otolaryngol Clin N Am 1996;29:377-392.

2. Balance $C A$. Some points in the surgery of the brain and its membranes. London: Macmillan 1907:249-284.

3. Sampath $\mathrm{P}, \mathrm{Holliday} \mathrm{MJ}, \mathrm{Brem} \mathrm{H}$, et al. Facial nerve injury in acoustic neuroma (vestibular schwannoma) surgery: etiology and prevention. J Neurosurg 1997;87:60-66.

4. Samii M, Matthies C. Management of 1000 vestibular schwannomas (acoustic neuromas): surgical management and results with an emphasis on complications and how to avoid them. Neurosurgery 1997;40:11-23.
5. Sterkers JM, Morrison GAJ, Sterkers O, et al. Preservation of facial, cochlear, and other nerve functions in acoustic neuroma treatment. Otolaryngol Head Neck Surg 1994;110:146-155.

6. Fenton JE, Chin RY, Fagan PA, et al. Predictive factors of long-term facial nerve function after vestibular schwannoma surgery. Otol Neurotol 2002;23:388-392.

7. Ikeda M, Abiko Y, Kukimoto N, et al. Clinical factors that influence the prognosis of facial nerve paralysis and the magnitudes of influence. Laryngoscope 2005;115:855-860.

8. Zaouche S, Ionescu E, Dubreuil C, et al. Pre and intraoperative predictive factors of facial palsy in vestibular schwannoma surgery. Acta Otolaryngol 2005;125:363-369.

9. Fenton JE, Chin RY, Shirazi A, et al. Prediction of postoperative facial nerve function in acoustic neuroma surgery. Clin Otolaryngol 1999;24:483-486.

10. Wiet RJ, Mamikoglu B, Odom L, et al. Long-term results of the first 500 cases of acoustic neuroma surgery. Otolaryngol Head Neck Surg 2001;124:645-651.

11. House JW, Brackmann DE. Facial nerve grading system. Otolaryngol Head Neck Surg 1985;93:146-147.

12. Tankere F, Bernat I, Vitte E, et al. Hypoglossal-facial nerve anastomosis: dynamic insight into the cross-innervation phenomenon. Neurology 2003;61:693-695.

13. Cross T, Sheard CE, Garrud P, et al. Impact of facial paralysis on patients with acoustic neuroma. Laryngoscope 2000;110:1539-1542.

14. Seiff SR, Carter SR. Facial nerve paralysis. Int Ophthalmol Clin 2002;42: 103-112.

15. Axon PR, Ramsden RT. Assessment of real-time clinical facial function during vestibular schwannoma resection. Laryngoscope 2000;110:1911-1915.

16. Darrouzet V, Martel J, Enée V, et al. Vestibular schwannoma surgery outcomes: our multidisciplinary experience in 400 cases over 17 years. Laryngoscope 2004;114:681-688.

17. Grayeli AB, Guindi S, Kalamarides M, et al. Four-channel electromyography of the facial nerve in vestibular schwannoma surgery: sensitivity and prognostic value for short-term facial function outcome. Otol Neurotol 2005;26:114-120.

18. Mamikoglu B, Wiet RJ, Esquivel CR. Translabyrinthine approach for the management of large and giant vestibular schwannomas. Otol Neurotol 2002;23:224-227.

19. Wu H, Sterkers JM. Translabyrinthine removal of large acoustic neuromas in young adults. Auris Nasus Larynx 2000;27:201-205.

20. Arts HA, Telian SA, El-Kashlan H, et al. Hearing preservation and facial nerve outcomes in vestibular schwannoma surgery: results using the middle cranial fossa approach. Otol Neurotol 2006;27:234-241.

21. Anderson DE, Leonetti J, Wind JJ, et al. Resection of large vestibular schwannomas: facial nerve preservation in the context of surgical approach and patient-assessed outcome. J Neurosurg 2005;102:643-649.

22. Catalano PJ, Post KD, Sen C, et al. Preoperative facial nerve studies predict paresis following cerebellopontine angle surgery. Am J Otol 1996;17:446-451.

23. McElveen JT, Belmont RG, Fukushima T, et al. A review of facial nerve outcome in 100 consecutive cases of acoustic tumor surgery. Laryngoscope 2000;110:1667-1672.

24. Kartush JM, Lundy LB. Facial nerve outcome in acoustic neuroma surgery. Otolaryngol Clin N Am 1992;5:623-647.

25. Sampath P, Rini D, Long DM. Microanatomical variations in the cerebellopontine angle associated with vestibular schwannomas (acoustic neuromas): a retrospective study of 1006 consecutive cases. J Neurosurgery 2000;92:70-78.

26. Ylikoski J. Pathological features of the facial nerve in patients with facial palsy of varying etiology. J Laryngol Otol 1990;104:294-300.

27. Kobayashi M, Tsunoda A, Komatsuzaki A, et al. Distance from acoustic neuroma to fundus and a postoperative facial palsy. Laryngoscope 2002;112:168-171.

28. Gormley WB, Sekhar LN, Wright DC, et al. Acoustic neuromas: results of current surgical management. Neurosurgery 1997;41:50-60.

29. Lanman TH, Brackmann DE, Hitselberger WE, et al. Report of 190 consecutive cases of large acoustic tumors (vestibular schwannoma) removed via the translabyrinthine approach. J Neurosurg 1999;90:617-623.

30. Neely JG, Neblett CR. Diferential facial nerve function in tumors of the internal auditory meatus. Ann Otol Rhinol Laryngol 1983;92:39-41. 